\title{
JUMP DIFFUSION MODELS FOR RISKY DEBTS: QUALITY SPREAD DIFFERENTIALS
}

\author{
HOI YING WONG \\ Department of Statistics, Chinese University of Hong Kong, \\ Shatin, Hong Kong, China \\ hywong@cuhk.edu.hk \\ YUE KUEN KWOK \\ Department of Mathematics, Hong Kong University of Science and Technology, \\ Clear Water Bay, Hong Kong, China \\ maykwok@ust.hk \\ Received 15 January 2003 \\ Revised 25 April 2003 \\ Accepted 30 April 2003
}

\begin{abstract}
The quality spread differential is defined to be the difference between the default premiums demanded for fixed rate and floating rate risky debts. The risky debt model based on Merton's firm value approach is used to examine the behaviors of the quality spread differential of fixed rate and floating rate debts. We extend earlier result by adopting Geometric Brownian diffusion process with jumps for the underlying firm value process of the debt issuer. Closed form formulas are obtained for the default premiums for risky debts. The impact of the jumps on the fixed-floating spread differential is examined.
\end{abstract}

Keywords: Risky debts; jump diffusion process; default premiums; quality spread differential.

\section{Introduction}

A corporate may raise capital by issuing either fixed rate or floating rate debts. In fixed rate debts, the par value of the debt paid by the issuer at maturity is fixed. A floating rate debt is similar to a money market account, where the par amount paid by the issuer at maturity is the sum of principal and accrued interests. The amount of accrued interests depends on the realization of the stochastic interest rate process over the life of the floating rate debt. It is a common practice for corporates to issue both fixed rate and floating rate debts. The preference of fixed rate over floating rate may signal the management view on possible rise of interest rate. In determining the appropriate proportion of debts into either fixed rate type or floating rate type, corporate executives consider financial attributes like balance sheet duration, current interest rate environment, and peer group practices [1]. 
Investors on a risky debt usually demand a default premium (also called credit yield spread), defined as the yield premium over the default free counterparts, in order to compensate for the potential loss due to the default of the debt issuer. In the fixed rate and floating rate debt markets, we would be interested to ask whether the default premiums demanded by investors are equal for both types of debts. A related query also appears in the determination of the swap rate for the fixed rate payer in an interest rate swap. Suppose the two counterparties in the swap contract differ in credit quality, does the swap rate depend on which party is serving as the fixed rate payer?

We define the quality spread differential to be the difference between the default premiums for fixed rate and floating rate risky debts. Empirical studies reveal that the default premiums for fixed rate debts are in general higher than those for floating rate debts. Various explanations to the positivity of quality spread differential based on arguments of market imperfections or agency cost have been proposed (see [2] for a summary of view points). Duffie and Liu [3] argued that if the term structure of the yield curve is upward sloping, floating rate coupons are likely to increase. Hence, floating rate debtholders should demand a higher floating spread, and this would lead to negative quality spread differential.

There are two most common approaches for analyzing credit risk of defaultable bonds. The reduced form or intensity based approach [4] uses the Poisson process to model default as sudden arrival. Such approach has been adopted by Duffie and Liu [3] to analyze the quality spread differential. The other approach, called firm value or structural model, attempts to describe the creditworthiness of the bond issuer by the firm value process of the issuer. Default occurs only if the firm value at bond's maturity falls short of obligations [5].

Copper and Mellon [6] initiated the analysis of quality spread differential using the Merton firm value model. They assumed constant interest rate for fixed rate debts and lognormal interest rate process for floating rate debts. Later, Ikeda [2] extended the risky debt models by assuming Vasicek mean reversion process for the interest rate. If the firm value process is a pure diffusion, then the firm never default unexpectedly since a sudden drop in firm value is impossible. Merton [7] initiated the modeling of asset price process by jump diffusion process in the pricing of options. Zhou [8] presented the economic rationales and implications for modeling firm value by jump diffusion. By employing a jump diffusion model for pricing corporate debt securities in a complex capital structure, Kijima and Suzuki [9] were able to capture realistic short maturity spreads observed in the market.

In this paper, we investigate the quality spread differential issue by assuming jump diffusion process for the firm value. We derive the closed form formulas for the default premiums for both fixed rate and floating rate debts. In particular, we would like to examine how the jumps in the firm value process affect the quality spread differential.

The following assumptions are made for the stochastic processes for the firm value and interest rate: 
(1) The firm value $V_{t}$ of the issuer follows the Geometric Brownian motion with random jumps. The governing stochastic differential equation for $V_{t}$ is given by

$$
d V_{t}=(\alpha-h v) V_{t} d t+\sigma V_{t} d W_{t}+V_{t^{-}} U d N_{t}
$$

and upon integration, we obtain

$$
\ln V_{T}-\ln V_{t}=\left(\alpha-h v-\frac{\sigma^{2}}{2}\right)(T-t)+\sigma\left(W_{T}-W_{t}\right)+\sum_{j=0}^{N_{T}-N_{t}} \ln \left(1+U_{j}\right) .
$$

Here, $\alpha$ is the instantaneous expected return of the firm value, $h$ is the mean number of Poisson arrivals per unit time. The jump amplitude $1+U$ is assumed to be lognormally distributed with mean $\ln (1+v)-\frac{\xi^{2}}{2}$ and variance $\xi^{2}$, and independent of $\left\{W_{t}\right\}_{t \geq 0}$ and $\left\{N_{t}\right\}_{t \geq 0}$. The jump component is the sum of Poisson distributed (with Poisson arrival rate $h$ ) $N_{T}-N_{t}$ iid random shocks. All parameters are assumed to be constant.

(2) The interest rate $r_{t}$ follows the Vasicek mean reversion process [10]

$$
d r_{t}=a\left(b-r_{t}\right) d t+\beta d Z_{t}
$$

where $d W_{t}$ and $d Z_{t}$ are correlated with $d W_{t} d Z_{t}=\rho d t$. Here, $a$ and $b$ denote the rate of reversion level and mean reversion level, respectively, and $\beta$ is the volatility of interest rate process.

Let $P(t, T)$ denote the price of the default free zero coupon. It can be shown that

$$
\frac{d P(t, T)}{P(t, T)}=r_{t} d t+\sigma_{P}(t, T) d Z_{t}
$$

where

$$
\sigma_{P}(t, T)=\beta \int_{t}^{T} \exp \left(-\int_{t}^{u} a d s\right) d u
$$

Merton's risky debt model

We adopt the formulation of Merton's risky debt model [5]. The Merton model assumes a simple capital structure of the firm, where the liabilities of the firm consist only of a single debt. The debt has zero coupon and no embedded option features. Default can be triggered only at maturity and this occurs when the firm value cannot meet its obligation. Accordingly, the payoff to the bondholders at maturity $T$ is given by

$$
B_{T}=\max \left(V_{T}, F_{T}\right)=F_{T}-\max \left(F_{T}-V_{T}, 0\right)=V_{T}-\max \left(V_{T}-F_{T}, 0\right),
$$

where $F_{T}$ is the par amount paid at maturity. The potential loss due to the default of the issuer can be visualized as the value of a put option on the firm value with strike price $F_{T}$. 


\section{Default Premium of Fixed Rate Debts}

Consider a fixed rate debt whose fixed par value at maturity $T$ is $F_{X}$. The payoff to the bondholders is given by $V_{T}-\max \left(V_{T}-F_{X}, 0\right)$. Let $B_{X}$ denote the price of the risky fixed rate debt at the current time $t$. By the Black-Scholes-Merton option pricing theory, the debt price is given by the discounted expectation of the terminal payoff:

$$
\begin{aligned}
B_{X} & =E_{Q^{t}}^{r, V, N}\left\{e^{-\int_{t}^{T} r_{s} d s}\left[V_{T}-\max \left(V_{T}-F_{X}, 0\right)\right]\right\} \\
& =V_{t}-P(t, T) E_{Q^{T}}^{r, V, N}\left\{\max \left(V_{T}-F_{X}, 0\right)\right\} .
\end{aligned}
$$

The last line indicates that the expectation is taken under the forward measure $Q^{T}$, where the change of measure is effected by the Girsanov Theorem. It may be convenient to use the default free zero coupon bond price $P(t, T)$ as the numeraire. Accordingly, we define $S_{t}=V_{t} / P(t, T)$ so that

$$
\frac{d S_{t}}{S_{t}}=-h v d t+\sigma_{S}(t, T) d \tilde{W}_{t}+U d N_{t} .
$$

Upon integration, we obtain

$$
\ln \frac{S_{T}}{S_{t}}=-h v \tau-\frac{\sigma_{S}^{2}}{2}+\int_{t}^{T} \sigma_{S}(u, T) d \tilde{W}_{u}+\sum_{j=0}^{N_{T}-N_{t}} \ln \left(1+U_{j}\right)
$$

where $\tau=T-t$ and

$$
\sigma_{S}^{2}(t, T)=\int_{t}^{T}\left[\sigma_{P}^{2}(u, T)+2 \rho \sigma \sigma_{P}(u, T)+\sigma^{2}\right] d u .
$$

We observe that conditional on $N_{T}-N_{t}=n, \ln \frac{S_{T}}{S_{t}}$ is the sum of $n+1$ independent normal distributions with conditional mean $-h v \tau-\frac{\sigma_{S}^{2}}{2}+n\left[\ln (1+v)-\frac{\xi^{2}}{2}\right]$ and conditional variance $\sigma_{S}^{2}+n \xi^{2}$. The conditional mean may be viewed as the dividend yield of $S_{t}$, conditional on $N_{T}-N_{t}=n$. By following a similar technique in [7], we then have

$$
\begin{aligned}
E_{Q^{t}}^{r, V, N}\left\{\max \left(V_{T}-F_{X}, 0\right)\right\} & =E^{N}\{P(t, T)\} E_{Q^{T}}^{r, V}\left\{\max \left(V_{T}-F_{X}, 0\right)\right\} \\
& =\sum_{n=0}^{\infty} \frac{h^{n} \tau^{n}}{n !} P(t, T) E_{Q^{T}}^{r, V}\left\{\max \left(V_{T}-F_{X}, 0\right) \mid n\right\} .
\end{aligned}
$$

If we define $P_{n}(t, T)=P(t, T)(1+v)^{-n} \exp (h v \tau)$ and use $P_{n}(t, T)$ as the numeraire, then

$$
P_{n}(t, T) E_{Q^{T}}^{r, V}\left\{\max \left(V_{T}-F_{X}, 0\right) \mid n\right\}=C_{E}\left(V, P_{n}(t, T) ; F_{X}\right)
$$

where $C_{E}\left(V, P_{n}(t, T) ; F_{X}\right)$ is the Black-Scholes European call price formula with discount factor $P_{n}(t, T)$ and strike price $F_{X}$. Combining the results together, we obtain

$B_{X}=\sum_{n=0}^{\infty} \frac{h^{n}(1+v)^{n} \exp (-h(1+v) \tau)}{n !}\left[V_{t} N\left(-h_{X}^{n}\right)+P_{n}(t, T) F_{X} N\left(h_{X}^{n}-\sigma_{X}^{n}\right)\right]$, 
where

$$
\begin{aligned}
h_{X}^{n}= & \frac{-\ln k_{X}+h v \tau-n \ln (1+v)}{\sigma_{X}^{n}}+\frac{\sigma_{X}^{n}}{2}, \quad k_{X}=\frac{P(t, T) F_{X}}{V_{t}}, \\
\left(\sigma_{X}^{n}\right)^{2}= & \sigma^{2} \tau+\left[\tau-2 \frac{1-e^{-a \tau}}{a}+\frac{1-e^{-2 a \tau}}{2 a}\right]\left(\frac{\beta}{a}\right)^{2} \\
& +\frac{2 \rho \beta \sigma\left(\tau-\frac{1-e^{a \tau}}{a}\right)}{a}+n \xi^{2} .
\end{aligned}
$$

Lastly, the default premium $\pi_{X}$ of the risky fixed rate debt is found to be

$$
\begin{aligned}
\pi_{X}= & \frac{1}{T} \ln \frac{F_{X}}{B_{X}}-\frac{\ln P(t, T)}{T} \\
= & -\frac{1}{T} \ln \left(\sum_{n=0}^{\infty} \frac{[h(1+v)]^{n} \exp (-h(1+v) \tau)}{n !} \frac{N\left(-h_{X}^{n}\right)}{k_{X}}\right. \\
& \left.+\frac{h^{n} e^{-h \tau}}{n !} N\left(h_{X}^{n}-\sigma_{X}^{n}\right)\right)
\end{aligned}
$$

\section{Default Premium of Floating Rate Debts}

The terminal payoff of a zero coupon floating rate bond is given by

$$
F_{T}=D e^{\int_{0}^{T} r_{s} d s}
$$

where $D$ is a fixed value specified in the bond indenture. We define the stochastic state variable $Y_{t}=\exp \left(\int_{0}^{t} r_{s} d s\right)$, which is the money market account, and $Y_{t}$ satisfies $d Y_{t}=r_{t} Y_{t} d t$. Suppose we choose $Y_{t}$ to be the numeraire and define $M_{t}=V_{t} / Y_{t}$. The process of $M_{t}$ under the risk neutral measure is found to be

$$
d M_{t}=-h v M_{t} d t+\sigma M_{t} d W_{t}+M_{t^{-}} U d N_{t},
$$

which is a martingale under the risk adjusted probability measure. The price of the risky zero coupon floating rate debt is given by

$$
\begin{aligned}
B_{L} & =V_{t}-E^{*}\left[\max \left(M_{T}-D, 0\right)\right] \\
& =V_{t}-\sum_{n=0}^{\infty} \frac{(h \tau)^{n} e^{-h \tau}}{n !} E^{*}\left[\max \left(M_{T}-D, 0\right) \mid n\right],
\end{aligned}
$$

where $E^{*}$ is the expectation under the risk adjusted measure. By following similar procedure as in the above fixed rate debt calculations, we obtain

$$
B_{L}=\sum_{n=0}^{\infty} \frac{(h \tau)^{n} e^{-h \tau}}{n !}\left[V_{t}(1+v)^{n} e^{-h v \tau} N\left(-h_{L}^{n}\right)+D N\left(h_{L}^{n}-\sigma_{L}^{n}\right)\right]
$$


where

$h_{L}^{n}=\frac{-\ln k_{L}+h v \tau-n \ln (1+v)}{\sigma_{L}^{n}}+\frac{\sigma_{L}^{n}}{2}, \quad k_{L}=\frac{D}{V_{t}} \quad$ and $\quad\left(\sigma_{L}^{n}\right)^{2}=\sigma^{2} \tau+n \xi^{2}$.

The default premium $\pi_{L}$ of the risky floating rate debt is found to be

$$
\begin{aligned}
\pi_{L} & =\frac{1}{T} \ln \frac{F_{T}}{B_{L}}-\frac{1}{T} \int_{0}^{T} r_{s} d s \\
& =-\frac{1}{T} \ln \left(\sum_{n=0}^{\infty} \frac{h^{n} e^{-h \tau}}{n !}\left[\frac{N\left(-h_{L}^{n}\right)}{k_{L}^{n}}+N\left(h_{L}^{n}-\sigma_{L}^{n}\right)\right]\right),
\end{aligned}
$$

where $k_{L}^{n}=D /\left[V(1+v)^{n} e^{-h v \tau}\right]$ is the jump adjusted quasi-debt ratio. Note that $\pi_{L}$ is independent of the default free bond price $P(t, T)$. Also, the volatility terms in $\pi_{L}$ depend only on the firm value volatility, but independent of the correlation coefficient $\rho$ and interest rate volatility $\beta$.

\section{Behaviors of the Quality Spread Differentials}

In order to compare the default premiums demanded for different types of debts, we consider an investor who has either means to raise the same amount of capital $B$. The par value $F_{X}$ in the fixed rate debt and the constant value $D$ in the floating rate debt are determined by solving the algebraic equations:

$$
B=B_{X}\left(V_{t} ; F_{X}\right) \text { and } B=B_{L}\left(V_{t} ; D\right) .
$$

Let $F_{X}^{*}$ and $D^{*}$ denote the solutions to the above algebraic equations. The fixedfloating differential is then given by

$$
D I F=\left.\pi_{X}\right|_{B_{X}=B}-\left.\pi_{L}\right|_{B_{L}=B}=\frac{1}{T} \ln \frac{P F_{X}^{*}}{D^{*}} .
$$

We would like to examine the impact of the arrival rate $h$ of the jumps and the variance $\xi$ of the jump amplitude on the fixed-floating quality spread differential. In Fig. 1, we plot the fixed-floating differential against bond maturity at varying level of Poisson arrival rate $h$. Other parameter values used in the calculations are $a=0.1, b=0.4, \beta=0.01, \sigma=0.2, V_{t}=1.0, \rho=-1, \xi=0.1$ and $v=0$. Here, we choose $\rho$ to be -1 so that the impact of jump frequency and jump amplitude on the fixed-floating differential can be revealed more clearly. Otherwise, the quality spread differential will be dominated by the correlation coefficient. We observe that the differential assumes negative values for medium life bonds ( 2 to 8 years) but changes sign for longer life bonds. As expected, the differential increases in magnitude when the frequency of arrivals becomes higher.

We also examine the impact of the variance $\xi$ of the jump amplitude (see Fig. 2). The same set of parameter values are used as before, except now $h$ is fixed at 0.1 . Similarly, the differential is seen to increase with increasing level of variance. To explain the phenomena as exhibited in the two figures, we examine the conditional 


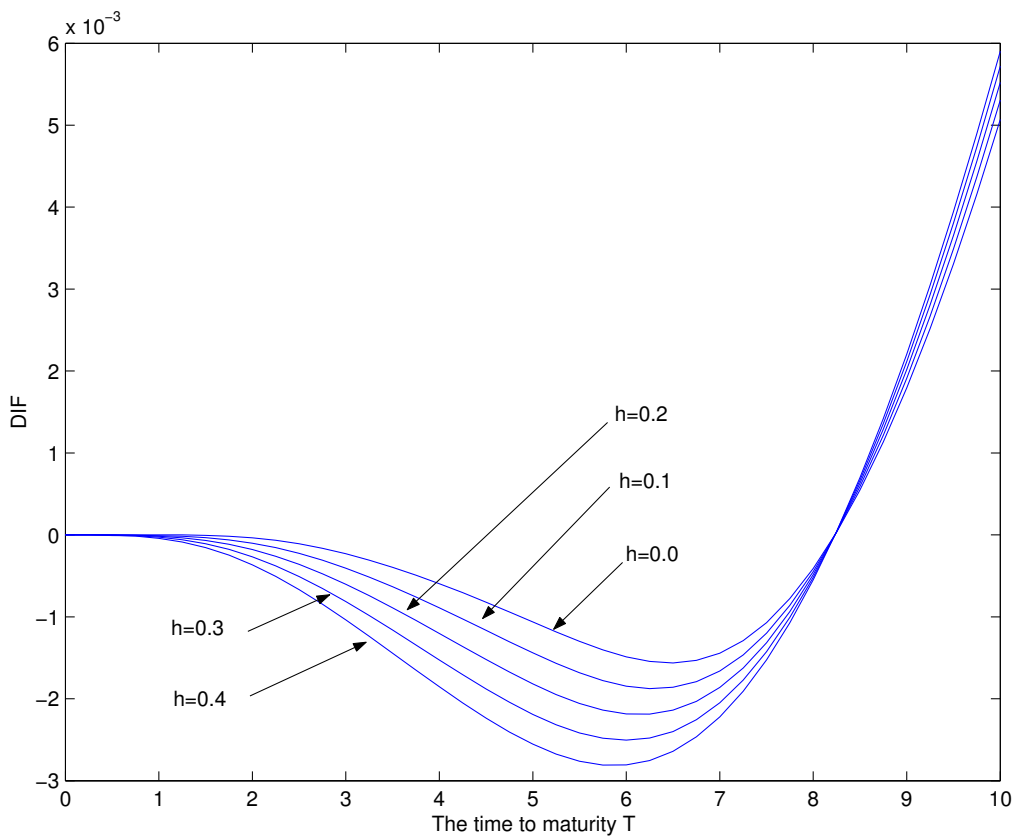

Fig. 1. Plot of fixed-floating differential against bond maturity at varying level of Poisson arrival rate $h$.

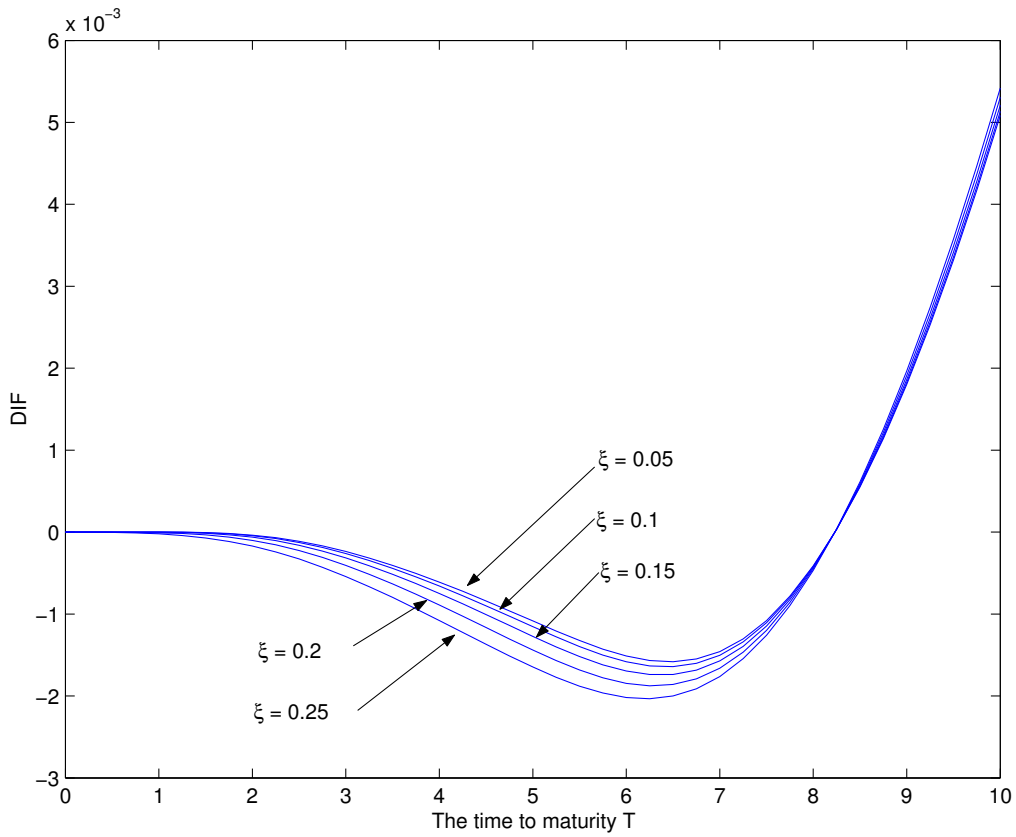

Fig. 2. Plot of fixed-floating differential against bond maturity at varying level of variance $\xi$ of the jump amplitude. 
effective variance of the fixed rate debt value and floating rate debt value and observe that the increase of jump frequency and amplitude would increase both bond values. When the correlation coefficient between the firm value and interest rate is negative, the impact of the jump frequency and amplitude is more profound on the floating rate debt value than the fixed rate counterparts for debts with medium maturities.

\section{Conclusion}

We have used the Merton firm value model for risky debts to examine the quality spread differential between fixed rate and floating rate debts. We assume that the firm value process of the issuer follows the Geometric Brownian process with jumps and the interest rate follows the Vasicek mean reversion process. By extending the analytic tools developed earlier by Merton, we are able to derive price formulas for the default premiums of the two types of debts. Our results illustrate that the impact of jumps in the underlying firm value process on the quality spread differential is more profound in medium life debts. In the future works, we may use more refined risky debt models that allow intertemporal default of the issuer and jumps in the stochastic interest rate process, and examine how these factors affect the quality spread differentials.

\section{References}

[1] C. Brobst and S. Huang, Fixed or floating, Risk (March Supplement, 2002) S16-S17.

[2] M. Ikeda, Default premiums and quality spread differentials in a stochastic interest rate economy, Advances in Futures and Options Research 8 (1995) 175-201.

[3] D. Duffie and J. Liu, Floating-fixed credit spreads, Financial Analysts Journal 73 (May/June 2001) 76-87.

[4] D. Duffie and K. J. Singleton, Modeling term structure of defaultable bonds, Review of Financial Studies 12 (1999) 687-720.

[5] R. C. Merton, On the pricing of corporate debt: The risk structure of interest rates, Journal of Finance 29 (1974) 449-470.

[6] I. A. Cooper and A. S. Mello, Default spreads in the fixed and in the floating interest rate markets: A contingent claims approach, Advances in Futures and Options Research 3 (1988) 645-665.

[7] R. C. Merton, Option pricing when underlying stock returns are discontinuous, Journal of Financial Economics 3 (1976) 125-144.

[8] C. Zhou, A jump-diffusion approach to modeling credit risk and valuing defaultable securities, Financial and Economic Discussion Series (1997-15), The Federal Reserve Board.

[9] M. Kijima and T. Suzuki, A jump-diffusion model for pricing corporate debt securities in a complex capital structure, Quantitative Finance 1 (2001) 1-10.

[10] O. Vasicek, An equilibrium characterization of the term structure, Journal of Financial Economics 5 (1977) 177-188. 